\title{
SPRO: SECURITY PROCESS FRAMEWORK
}

\author{
Henrique Michel Persch, Lisandra M. Fontoura \\ Departamento de Computação Aplicada (DCOM) \\ Universidade Federal de Santa Maria (UFSM) \\ Santa Maria, Brasil \\ \{hpersch, lisandra\}@gmail.com
}

\author{
Adriano Brum Fontoura \\ Instituto Federal Farroupilha \\ Santa Maria, RS, Brasil \\ fontoura.ab@gmail.com
}

\begin{abstract}
Secure software implies that the process used in its development includes activities to insert, monitor and ensure security from the early stages of the software process. This article proposes the Security Process Framework that aims to facilitate the task of creating secure processes through the reuse of process components developed from security patterns. The components are recovered and prioritized from a repository through multicriteria techniques that consider security requirements and characteristics related to the project context. Software process lines are used to organize the selected components and to assemble the secure software process. Furthermore, a tool called SPro System was developed to support the use of the framework. Case studies were used to verify the applicability of the proposal, which showed that the framework and SPro System) facilitated the tailoring and decreased the time spent in the definition of security processes.
\end{abstract}

Keywords - security patterns, security process, software process lines, process tailoring

\section{INTRODUCTION}

Nowadays, software are becoming increasingly complex, requiring high levels of security to prevent the occurrence of failures and data loss. Several authors propose that methodologies and processes for software development should be concerned with the security from the early stages of projects life cycle [8][9].

Nevertheless, to create software processes aimed at a secure software development is a long process, time consuming and that requires many resources. In addition, the processes must be suited to the needs of each project, which requires defining activities and security practices appropriate to the context and that includes the security requirements defined for the project [3][4][18]. This task is not trivial and it requires experts in security and in software processes, which are not always available in the team.

In the literature, numerous security standards and models are presented which describe best practices and security requirements, such as: ISO/IEC 27001 [1], ISO/IEC 27002 [2] and ISO/IEC 21827 [10]. However, these standards, as any other standard, do not detail the processes that need to be followed to implement security in its projects.

To facilitate the task of setting secure software processes, this paper proposes a framework consisting of: (1) a repository of process components defined from the security patterns; (2) techniques for prioritization and selection of these components from context information; (3) process lines that organize

DOI reference number: 10.18293/SEKE2019-015 process components contributing to their reuse and (4) a tool to support the use of the framework.

The components stored in the repository were defined from security patterns catalogs [15], security standards [1], [10] and scientific literature. Security patterns provide solutions already established to recurring security problems and serve as a reference for organizations that seek to meet security requirements [15]. Process components facilitate the development of new processes through the reuse of predefined components and enable the assessment and improvement of these through the use in different software projects. The components are associated with security requirements through rules and are prioritized from multicriteria techniques that consider the context of the project. The prioritized components are arranged using software process lines.

This article is organized as follows: in Section 2, related studies are discussed; in Section 3, concepts relevant to the understanding of the study are introduced; in Section 4, the proposed framework is described showing how the components of the processes and the prioritization methods were defined; in Section 5, the support tool is explained; and in Section 6, a case study is presented. Finally, in Section 7, the final considerations of the study are described.

\section{RELATED WORK}

In [11], a methodology for adapting software processes is presented, based on security requirements recommended by the security practices of the ISO/IEC 21827. The processes are compiled from components of the Rational Unified Process (RUP), Extreme Programming (XP) and on security patterns proposed in the literature. This study was developed in the same research group and the main differences is that it does not consider the context of the project, the use of prioritization techniques and software process lines.

Mellado, Medina and Piattini [6] propose to incorporate security requirements from the early stages of development using software product lines. Thus, the authors seek to facilitate the compliance with security standards and to manage potential variabilities that may happen among security requirements. The authors propose the use of security standards (ISO/IEC $27001 \mathrm{e}$ ISO/IEC 15408) to manage security requirements. The focus of the work is on the product and our work focuses on the process.

In [7], the authors propose an extension of the Scrum software development framework with features focused on creating secure software.

Hamid and Weber [14] propose a model-driven engineering (MDE) methodological approach associated with a pattern- 
based approach to support the development of secure software systems.

However, this study differs from the others due to the development of a secure processes tailoring framework using process components associated with the process area from ISO/IEC 21827. The process components are prioritized from multi-criteria decision techniques that consider context information. In addition, software process lines are used which organize the recovered components to form the process and a support tool is provided.

\section{BACKGROUND}

Security standards and models have key goals and practices so that organizations can define the expected level of security in their processes. In the literature, various standards of information security are described, among which we can highlight: ISO/IEC 27001, ISO/IEC 15408 and ISO/IEC 27002. ISO/IEC 27001 [1] describes a process for information security management in an organization that structures activities through a continuous improvement cycle. On the other hand, ISO/IEC 15408 provides a common set of requirements for security functions in products and systems and for assurance of measures applied to them during a security assessment [12]. The model SSE-CMM - Systems Security Engineering Capability Maturity Model, published as ISO/IEC 21827: 2008 [10], describes a set of process areas (PAs) that are required in a security engineering process. ISO/IEC 21827 proposes 22 process areas (PAs) that are organized into two groups: Security Base Practices and Project and Organizational Base Practices [10]. For each PA, it is presented a list of BPs (Base Practices) that assist in meeting the goals of the process areas (PA).

Security standards and models describe ways that are intended to assist in the development of security processes, providing practices to be implemented or guidelines to be followed by organizations. In this study, we chose to use the ISO/IEC 21827 [10] because this model defines a set of best practices that guide the organization in the implementation of effective security processes. In addition, this model is widely known and widespread.

Nevertheless, the ISO/IEC 21827, as well as other standards, does not detail the activities to be implemented by organizations. Therefore, it was decided to seek solutions in the literature used by other organizations that have been successful. Many of these solutions in the security area are documented as security patterns [15]. Even though many security patterns and techniques to use them are being proposed, it is complex to adapt and integrate them in every stage of the software development or in specific contexts.

In this study, the patterns are described as process components and associated with a specific use context. However, describe components and store them in a repository does not guarantee the proper selection and integration of these components to create a consistent software process. To solve this issue, the process components are selected through multicriteria techniques and organized using software process lines (SPrL). SPrL are intended to represent the dynamic aspect of the processes and a metamodel is used to describe the structural aspect of the components.

Software process lines emerged from software product lines and are aimed at the development of consistent processes, enabling the reuse of previously defined components. SPrL is a form of tailoring of processes that has the following objectives: i) increase the quality and adequacy of the processes; ii) representation of variabilities and similarities among processes to maximize reuse; and iii) reduce the risks of an inadequate tailoring of process [16].

The multi-criteria techniques help the decision making from the analysis of preferred criteria necessary for understanding the reality of the analyzed problem and the choice of the alternative that will allow the best decision to be made. These are examples of multi-criteria methods: AHP (Analytic Hierarchy Process) [19], TODIM (an acronym in Portuguese for Iterative and Multi-criteria Decision Making) [13], and TOPSIS (Technique for Order of Preference by Similarity to Ideal Solution) [5].

The theoretical foundation of the AHP is based on the decomposition and synthesis of the relations among the criteria until a prioritization of their indicators is reached, approaching a better response of single measurement of performance [19]. The TODIM is based on the Prospect Theory [13]. On the other hand, the TOPSIS is based on the calculation of the Euclidean distance between the most beneficial alternative and the costlier alternatives [5].

In our study, the prioritization methods are used to select the most appropriate process components for the project in question, using criteria that define the context. Several authors propose that the context should be considered for definition of software processes, such as: Boehm and Turner [4], Cockburn [3] and Kruchten [18]. In this study, we chose to use the context described by the Octopus Model, as proposed by Kruchten [18], which uses the following contextual factors: size, stable architecture, business model, team distribution, rate of change, system age, criticality and governance.

\section{SECURITY PROCESS FRAMEWORK}

This section describes the Security Process Framework through detailing the process components repository and metamodel, the rules of components association to security requirements and the techniques used for the prioritization of the components.

\section{A. Repository of Security Processes}

The process components were defined from classes described in the metamodel (Figure 1), elaborated according to Software and Systems Process Engineering Metamodel Specification (SPEM) [17].

A process component is an aggregation of tasks that are performed by roles which have artifacts as input and output and are associated with a discipline. Furthermore, project processes are composed of process components and are associated with projects that use the process. 


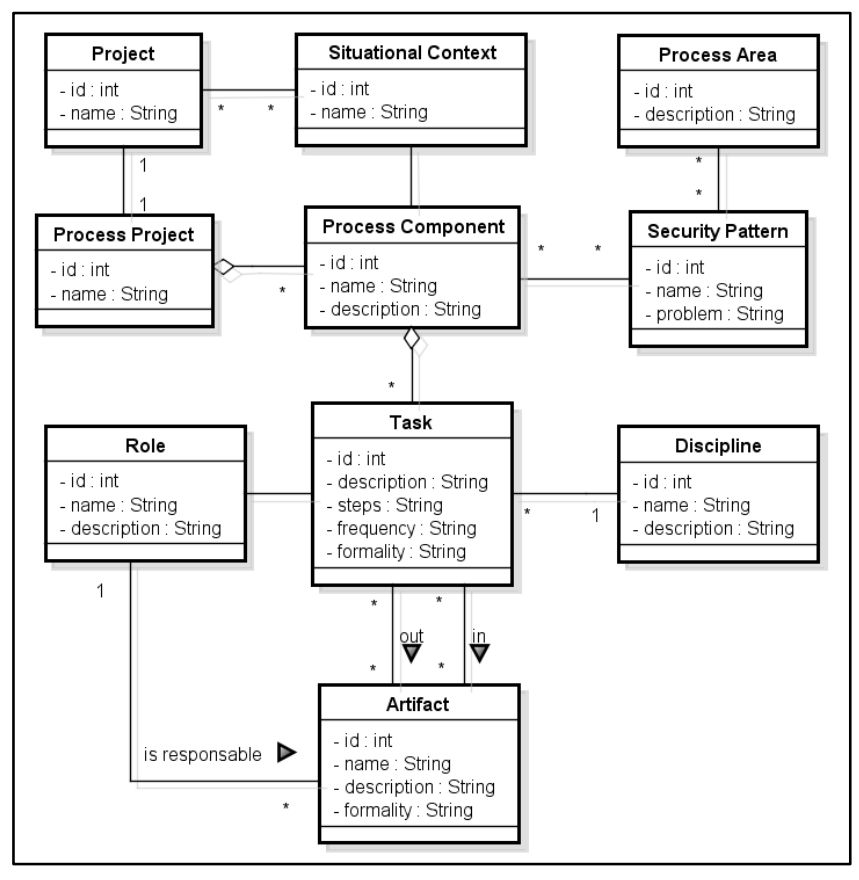

Figure 1. Security Process Metamodel

Process components and projects are associated with information of the situational context. Process components are associated with security patterns from which they were defined. Security patterns [15] are associated with process areas that they meet, extracted from the ISO/IEC 21827 [10].

To illustrate how the classes described in the metamodel are used to define the components, Figure 2 shows the process component Asset Valuation, described from the pattern of the same name [15]. This pattern aims at helping the organization to determine the overall importance of the assets under its control and ownership.

\section{B. Security Components Association to PAs}

The defined process components are associated with the goals of the 11 PAs - security base practices, found in the standard ISO/IEC 21827 [10]. For example, the component Asset Valuation (Figure 2) was associated with the process area PA 03 - Security Risks Assessment.

After the analysis of several catalogs of security patterns, each security pattern was described as a process component and associated with one or more process areas that it covers. These components were placed in a repository and are available for use in the development of processes that aim to meet security requirements. Thenceforth, it was possible to build a repository of software process components based on the ISO/IEC 21827 [10], with 50 process components, which have about 70 artifacts and 60 tasks.

\section{Determination of the Prioritization Methods}

The multi-criteria methods are used to select process components that have a context of use similar to the project context. These contexts are described using the attributes proposed by the Octopus Model.

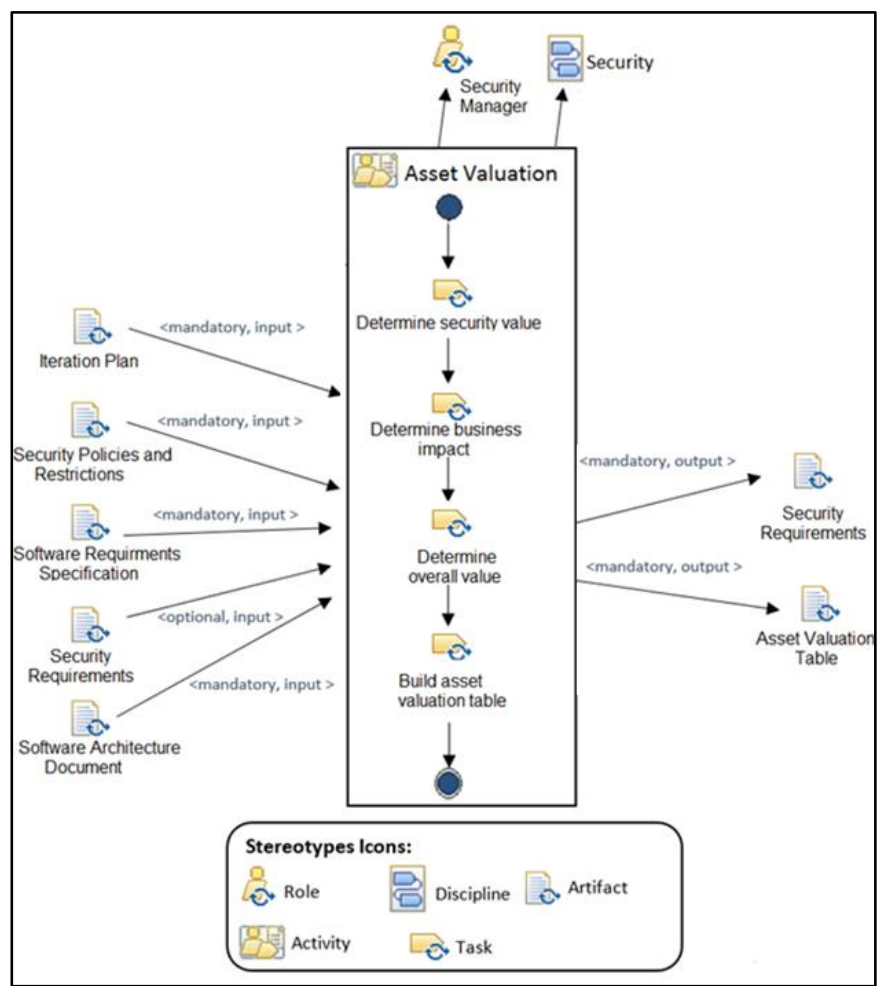

Figure 2. Details of the process component Asset Valuation

In Figure 3, the process area PA08 - Assess Security Risk is shown associated with four components of different processes that aim at satisfying it. The contexts of the component and of the project are assessed and compared according to the calculations proposed by each prioritization method to assist in choosing the best components.

\section{SPRO SYSTEM}

In this section, a tool that supports the use of the framework for tailoring of processes, using software process lines, called SPro System is described. Process components defined from RUP and XP were inserted into the repository. Process lines have been defined to organize the components and architectures to represent the variants.

The processes tailoring are performed through four stages, which are: i) definition of the project context; ii) selection of the tailoring requirements and of the processes architecture; iii) prioritization of activities; and iv) creation of the tailored process. To illustrate the stages, the implementation of an example of use is described.

The situational context of the project is defined in order to select the most appropriate process components for the project. This example refers to a new development in a well-known domain that involves only loss of money and it is being developed by a local team of 25 people, the values were defined as follows: size $=$ medium, change of rate $=$ less than 10, type of architecture $=$ new, age of the system $=$ new development, business model $=$ commercial, criticality $=$ loss of money, distribution of the team $=$ local and governance $=$ simple rules. 


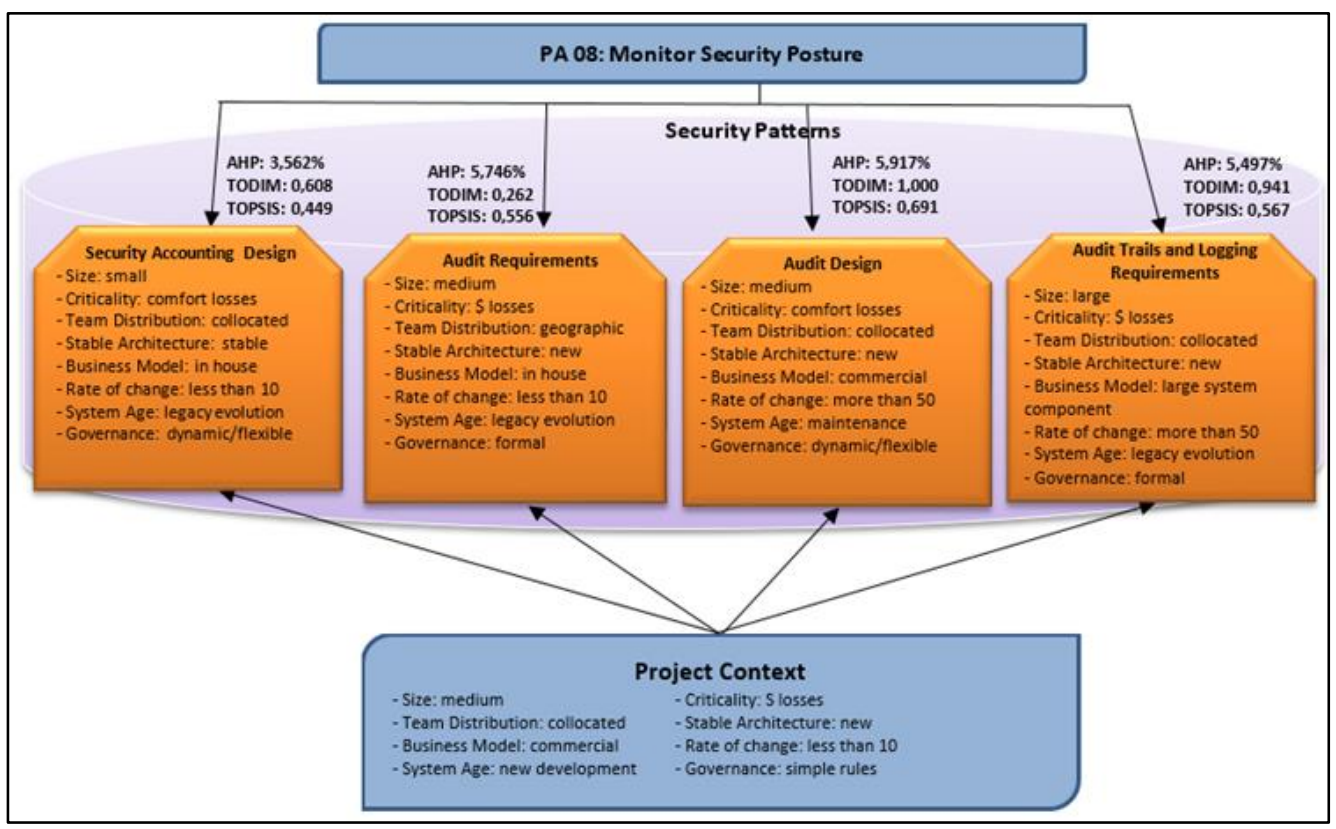

Figure 3. Selection of the Process Patterns

In the second stage, the process engineer selects the tailoring criteria and the architecture. For the tailoring criteria, the PAs 03, 05, 07 08, 10 were selected as showed in Figure 4 (A). In the tool, different tailoring criteria can be used, such as risks, quality, however, this article is limited to describe on tailoring criteria based on the ISO/IEC 21827.

For each selected tailoring criterion, the SPro System searches in the repository the process components that were previously associated with the tailoring criteria through rules, that is, the components that aim at meeting the tailoring criterion. For these components, the similarity between the context of the project and of the component is calculated. The similarity values generated by the application of multi-criteria techniques help process engineers' decision-making, however, the selection is their responsibility. The multi-criteria techniques are an important source of information because they are based on different methodologies of analysis, prioritizing the most relevant activities for the development in particular. Figure 4 (B) shows the results of the prioritization of the components. From the defined process architecture and the recovered process components, prioritized and selected, the specific process for the project was created (Figure $4 \mathrm{C}$ ).

\section{VALIDATION USING CASE STUdY}

For the validation, a case study was carried out, which was applied to a class of the Master's course in Computer Science from the Federal University of Santa Maria in the discipline of Software Process Improvement. The following materials were used for the experiment:

- A set of 11 cards with the name of the PAs proposed by the ISO/IEC 21827, which are the tailoring criteria to be selected;

- A set of 45 cards representing the process components, elaborated from the security patterns, contextualized according to the attributes proposed by the Octopus Model;

- Description of two scenarios of development of a software;

- Description with examples of associations of PAs with process components elaborated from the literature;

- Sheet of paper to be filled in by the group with the PAs and the process components selected for the project described in the scenario.

Initially, the students were divided into 2 groups with 4 members each. Each group received the support material described above. The group's objective was to develop a software development process from the scenario. To this end, they needed to identify which PAs should be selected in the described project and, then, select the appropriate process components to the PAs and to the context of the project. In the first stage, the experiment was performed without SPro System and in the second stage the SPro System was used. In each stage, a different scenario was used.

\section{A. Observed Results}

The most striking factor was the decrease in time to elaborate the process. The time spent in the first stage was about 1 hour and 15 minutes. In the second stage, the average time was 25 minutes, thus the SPro System helped the team to reduce the time by approximately $60 \%$.

Regarding the context of the project, the two groups correctly extracted the characteristics of each project from the provided scenario. According to the analysis performed in the process architectures developed in the first stage, it was possible to verify that group 1 was able to determine the exact process areas that encompassed the scenario. In contrast, group 2 included two extra process areas, which were not required by the scenario. 


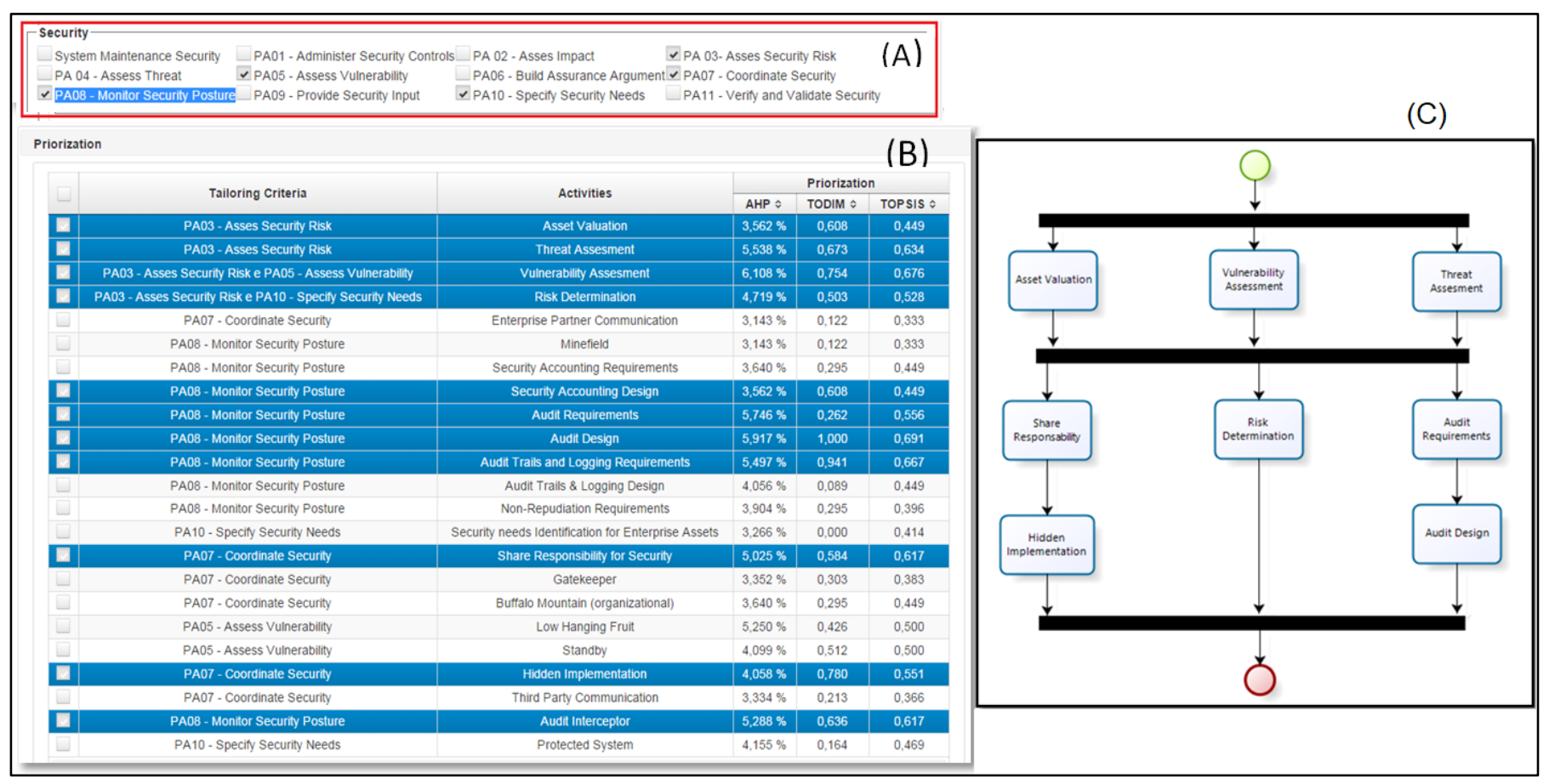

Figure 4. SPro System

Regarding the process components selected for each tailoring criterion, it was possible to verify that group 1 selected 10 activities for 5 tailoring criteria. Thus, it was verified that this group correctly compared the contexts of the activities and the project, besides an analysis of the description of the activity. Group 2 has determined 19 activities for 5 tailoring criteria, however, it was found that this group ignored the context and the activity characteristics, giving greater attention to the description of the activity. In this stage, both groups were able to determine the activities and the tailoring criteria considered as essential in the presented scenarios.

In the second stage, the activity was developed according to the first step, however using the SPro System and with another scenario. It is possible to state that the groups obtained more satisfactory and significant results when using the tool because they selected the activities and the tailoring criteria considered as essential in each presented scenarios

\section{B. $\quad$ Assessment Questionnaire}

To complement the analysis, a questionnaire was applied so that the students could assess the framework and the SPro System. The questionnaire responses are showed in the Figure 5.

All participants agreed that the use of the tool facilitated the processes tailoring aiming at meeting the security requirements (Q.4). Furthermore, they fully agreed on the importance of maintaining a repository with adapted process components to facilitate new adaptations (Q.8). Regarding the ease of understanding and the translation of the security patterns for the activities so that they can be added to a software process, there was a partial agreement (Q.2).
Only one participant partially disagreed that the provided patterns were sufficient to meet the tailoring criteria (Q3), wherein four answers partially agreed and the other 3 totally agreed. In this sense, it can be highlighted that the groups considered that the use of security patterns may be a viable solution to solve the security problems in the project, however, it may not be the only solution that should be applied.

The participants recognized that the pre-defined process components associated with the tailoring criteria facilitated the development of the process lines (Q.5). All participants agreed that the SPro System tool assists in the processes tailoring considering security requirements (Q.6).

Nonetheless, when asked if the tool expresses the stages required to define processes, there were different responses, wherein four participants fully agreed, three partially agreed and one neither agreed nor disagreed (Q.7). From these responses, there was the need to improve the stages to be used in the tool for the processes tailoring. In addition to this improvement, in the descriptive responses, suggestions regarding the SPro System were given.

Moreover, in the space for comments, the participants reported the difficulty in detecting security patterns and activities that meet the tailoring criteria.

Regarding the prioritization methods, it was possible to verify that the groups thoroughly analyzed the numbers obtained and brought in the calculations made by the AHP, TODIM and TOPSIS methods. According to the participants, the methods were an excellent source of information and guided the groups in the decision-making regarding which process components they should select. 


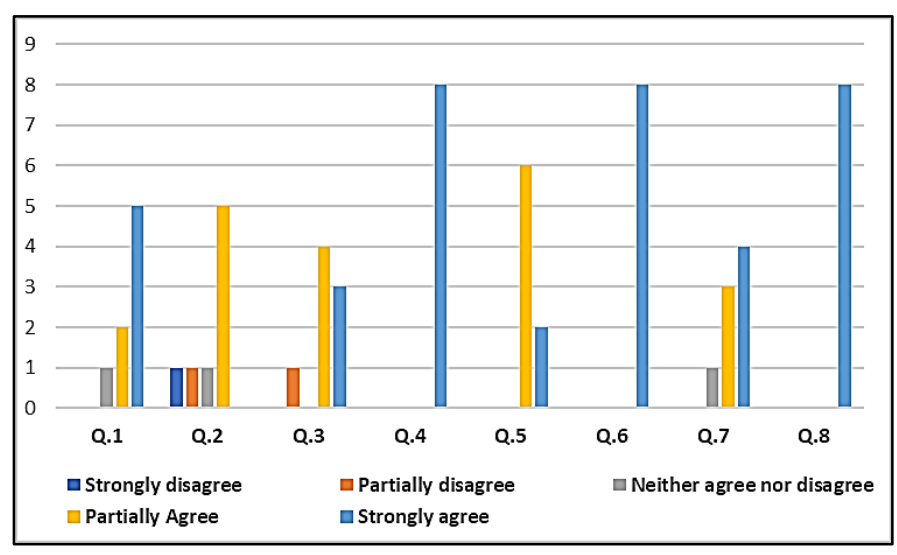

Figure 5. Responses of the applied questionnaires

From the development of this case study, it was possible to evaluate the use of the SPro System tool and to apply the concepts presented in the elaborated framework.

\section{FINAL CONSIDERATIONS}

This paper presented a framework proposal that aims at selecting process components that can be used for the tailoring of security processes. The process components were developed and derived from security patterns and were proven efficient in the development and tailoring using software process lines. Furthermore, with the use of multi-criteria techniques, the framework assists the process engineer to choose the most relevant components for the development process, considering the contexts of project and of the components. With the support of the SPro System tool, the use of the proposed framework was demonstrated.

The validation showed that the framework and the SPro System facilitated the process tailoring and decreased the time spent in the definition of security processes. In addition, the students, even without security expertise, were able to select activities appropriate to the scenario, properly creating the process according to the problem they received.

As future studies, we suggest the semi-automatic selection of process components and the expansion of the repository comprising other security standards.

\section{REFERENCES}

[1] —. 2013. ISO/IEC 27001:2013 - Information technology - Security techniques - Information security management systems - Requirements. 2013.

[2] - 2013. ISO/IEC 27002:2013. Information technology -- Security techniques -- Code of practice for information security controls. 2013.

[3] A. Cockburn. 2004. Crystal Clear: A Human-Powered Methodology for Small Teams. Addison-Wesley, Boston, 2004.

[4] B. Boehm, R. Turner. 2009. Balancing Agility and Discipline. Pearson Education, Inc, Boston, 2009.

[5] C. L. Hwang, K. Yoon. Multiple attribute decision making; methods and applications, In: Proc. Lecture
Series in Economics and Mathematical Systems, Berlin, Germany, Springer-Verlag, 1981.

[6] D. Mellado, E. Fernandez-Medina, M. Piattini. Security Requirements Variability for Software Product Lines. In: Third International Conference on Availability, Reliability and Security, 2008.

[7] C. Pohl, H. Hof. Secure Scrum: Development of Secure Software with Scrum. International Conference on Emerging Security Information, Systems and Technologies (SECURWARE) 2015, Venice, Italy, 2015.

[8] E. Amoroso, "Recent Progress in Software Security," in IEEE Software, vol. 35, no. 2, pp. 11-13, March/April 2018.

[9] S. HUSSAIN, G. RASOOL, M. ATEF, A. K. SHAHID. A Review of Approaches to Model Security into Software Systems, 2013. Journal of Basic and Applied Scientific Research, ISSN 2090-4304. Lahore, Pakistan.

[10] ISO/IEC 21827. Associação Brasileira de Normas Técnicas. NBR ISO/IEC 21827:2008 Information technology. Security techniques. Systems Security Engineering. Capability Maturity Model (SSE-CMM). Switerzland, 2008.

[11] R. Wagner, L. M. Fontoura, A. B. Fontoura. Using Security Patterns to Tailor Software Process. In: Proc. International Conference on Software Engineering and Knowledge Engineering (SEKE), 2011. pp. 672-677.

[12] ISO/IEC15408. ISO/IEC15408 - Information technology - Security techniques - Evaluation criteria for IT security. 2005.

[13] L. F. Gomes, M. P. Monica, L. A. D. Rangel. An application of the TODIM method to the multicriteria rental evaluation of residential properties. European Journal of Operational Research, 193, 204-211, 2009

[14] B. Hamid, D. Weber. Engineering secure systems: models, patterns and empirical validation. Computer \& Security. 77, 315-348 (2018).

[15] M. Schumacher, E. Fernandez-Buglioni, D. Hybertson, F. Buschmann, P. Sommerlad. Security Patterns Integrating Security and Systems Engineering. John Wiley \& Sons, 2013.

[16] O. Jaufman, J. Münch. Acquisition of a project-specific process. Springer, Berlin, Heidelberg, 2005. pp. 328-342.

[17] OMG. 2008. Software \& Systems Process Engineering Meta-Model Specification, Version 2.0. Software Engineering Institute, Carnegie, Pittsburgh, 2008.

[18] P. Kruchten. Contextualizing Agile Software Development. Vancouver, BC, Canada Proc. European System, Software \& Service Process Improvement \& Innovation (EuroSPI) 2010, 2010.

[19] T. L. Saaty, Theory and Applications of the Analytic Network Process: Decision Making with Benefits, Opportunities, Costs, and Risks. RWS Publications, Pittsburgh, 2005. 